\title{
Lateral lipid organization of the bovine milk fat globule membrane is revealed by washing processes
}

\author{
Haotian Zheng, ${ }^{*} \dagger \ddagger^{1}$ Rafael Jiménez-Flores, $\ddagger$ and David W. Everett ${ }^{*} \dagger$ \\ ${ }^{*}$ Riddet Institute, Palmerston North 4442, New Zealand \\ †Department of Food Science, University of Otago, Dunedin 9054, New Zealand \\ ‡Dairy Products Technology Center, California Polytechnic State University, San Luis Obispo 93407
}

\begin{abstract}
Evidence for the asymmetric distribution of phospholipids in the milk fat globule membrane (MFGM) was obtained by applying 3 washing processes using aqueous solutions with different degrees of stringency (mild, intermediate, and intensive) to milk fat globule (MFG) surfaces in simulated milk ultrafiltrate buffer. We detected no change in the amount of cholesterol after the mild washing process; however, intensive washing yielded a relative enrichment of surface cholesterol with concomitant damage to the outer bilayer of the MFGM. This finding supports the hypothesis of repartitioning of cholesterol on MFG surfaces during mechanical treatments. An updated model system of lipid organization was developed according to the results of relative depletion of individual phospholipids, as analyzed by HPLC.
\end{abstract}

Key words: milk fat globule membrane, washing process, lipid organization, cholesterol

\section{INTRODUCTION}

The milk fat globule (MFG) is enveloped and stabilized by a bioactive, multi-component layer referred to as the milk fat globule membrane (MFGM) with a thickness of around 10 to $20 \mathrm{~nm}$ (Walstra, 1999). This membrane regulates the physicochemical and nutritional functions of MFG (Michalski and Januel, 2006; Argov et al., 2008; Dewettinck et al., 2008). According to physiological secretion mechanisms of the MFG, the MFGM is constructed of phospholipids (PL), cholesterol, and membrane proteins (Keenan and Mather, 2006; MacGibbon and Taylor, 2006); however, to date, the composition and structure of the MFGM are not conclusively known.

Food is a complex arrangement of multiple components that are digested and absorbed though a dynamic and interrelated process, rather than as individual

Received January 15, 2014.

Accepted June 27, 2014

${ }^{1}$ Corresponding author: haotian.zheng@otago.ac.nz components, highlighting the importance of food microstructure in delivering health benefits. For instance, food structure may be designed to control digestion, release, and absorption of lipophilic food components (McClements et al., 2008). The biofunctionality of the MFGM is not simply a sum of those properties from individual molecules but is affected by the specific organization and structure of the PL, cholesterol, and membrane proteins. Although current models show differences in the organization of lipids and proteins (Michalski et al., 2002; Lopez et al., 2011; Mather, 2011), it is generally agreed that the MFG inner core (triacylglycerides) is coated with a trilayer MFGM system consisting of a primary monolayer of proteins and lipids and an outer PL bilayer with associated glycocalyx. Mather (2011) presented a topology of the major bovine MFGM proteins, where the location and specific interactions within the MFGM were shown. Lopez and coworkers (2011) examined MFGM lipid organization and suggested co-existence and phase separation between tightly packed liquid-ordered domains ( $\boldsymbol{L}_{\mathbf{o}}$, also known as lipid rafts in biological membranes) enriched in sphingomyelin (SM) and cholesterol, and liquid-disordered domains $\left(\boldsymbol{L}_{\mathrm{d}}\right)$ enriched in other glycerolphospholipids, such as phosphatidylcholine, phosphatidylethanolamine, phosphatidylinositol, and phosphatidylserine, with an asymmetric distribution of $L_{\mathrm{o}}$ in the outer leaflet of the outer PL bilayer.

Separation of MFG and subsequent washing to remove non-membrane-associated proteins is the first step to extract the MFGM (Mather, 2000). Methods have been reported utilizing different centrifugal forces, centrifugation times, and washing buffers (Huang and Kuksis, 1967; Patton and Huston, 1986; Ye et al., 2002; Le et al., 2009). It is widely accepted that mechanical treatments such as centrifugation induce depletion of MFGM materials from MFG (Walstra et al., 2006); therefore, it is reasonable to question whether the MFGM compositional data generated from different isolation methods can be compared.

Protein structural changes in the MFGM may be induced by centrifugal washing processes (Zheng et al., 2013), which can cause irreparable damage to the 
outer phospholipid layer of the MFGM and depletion of MFGM components. In this study, the relative compositional changes in lipids were measured after centrifugal washing processes. From these measurements, we proposed an updated MFGM structural model of lipid organization.

\section{MATERIALS AND METHODS}

\section{Materials}

Bovine raw milk samples were collected from the same Jersey cow in good health and in mid lactation (autumn) on a local farm (Port Chalmers, New Zealand). We sampled milk from one cow to avoid the problem of mixing milk from cows at different stages of lactation; to avoid the need for stirring and refrigerated storage of pooled milk, which would alter the MFG surface; and to avoid possible inconsistent pooling of milk from several cows. The cow was pasture-fed and vacuum-milked over 6 consecutive sampling days. No extra mechanical or thermal treatments were applied to the fresh raw milk before experiments. Simulated milk ultrafiltrate (SMUF) at $\mathrm{pH} 6.5$ (Jenness and Koops, 1962) was prepared and used as a buffer and washing solution. The following phospholipid standards were obtained: 3-sn-phosphatidylethanolamine (PE) and L- $\alpha$ phosphatidylcholine (PC), both from bovine brain; L- $\alpha$-phosphatidylinositol (PI) from bovine liver; 1,2-diacyl-sn-glycero-3-phospho-L-serine (PS) from bovine brain (Sigma-Aldrich, St. Louis, MO); and SM from bovine milk (Avanti Polar Lipids, Alabaster, AL). All other chemicals used were analytical grade and obtained from Merck (Darmstadt, Germany).

\section{Isolation of MFG}

Three centrifugal isolation methods: (1) $3,000 \times g$, 5 min, 3 washes (M1); (2) $3,750 \times g, 15$ min, 1 wash (M2); and (3) $15,000 \times g, 20 \mathrm{~min}, 3$ washes (M3) were used for collecting and washing MFG from raw milk according to previously published work (Patton and Huston, 1986; Ye et al., 2002; Gallier et al., 2010b) respectively, with minor modifications. Methods M1 and M3 were carried out by washing centrifuged raw milk 3 times in SMUF using either the M1 or M3 centrifugation process. The cream obtained from this initial centrifugation of raw milk was resuspended in 10 volumes of SMUF and left at ambient temperature for $1 \mathrm{~h}$, and then centrifuged again (using either the M1 or M3 process, as appropriate) to remove non-MFGM proteins from the MFG surface. This is referred to as the first wash (M1-1 and M3-3), and was repeated a further 2 times using M1 or M3 to obtain the second and third washed MFG suspensions (M1-2, M1-3; M32, M3-3). The M2 process (Patton and Huston, 1986) used a sucrose density gradient separation as a single wash process that was applied as a comparison method. In this method, $15 \mathrm{~mL}$ of sucrose-treated raw milk (containing $5 \% \mathrm{wt} /$ vol sucrose) was layered under $30 \mathrm{~mL}$ of SMUF buffer in centrifugation tubes, and washed MFG were collected after M2 centrifugation. A control MFG suspension sample from M2 was collected by direct centrifugation of sucrose-treated raw milk. The temperature of the SMUF buffer was $30^{\circ} \mathrm{C}$ and centrifugation temperature was $25^{\circ} \mathrm{C}$ for all experiments. Raw milk and MFG fraction samples, which were collected after each centrifugation process, were stored at $-80^{\circ} \mathrm{C}$ before further analysis.

\section{Size Distribution and Assessment of Specific Surface Area of MFG}

Volume-weighted mean diameter $\left(\boldsymbol{d}_{43}\right)$ and surface area-weighted mean diameter $\left(\boldsymbol{d}_{32}\right)$ were determined by laser diffraction using a Horiba particle analyzer LA-950 (Horiba, Irvine, CA). The refractive index of MFG was taken as 1.460 at $655 \mathrm{~nm}$ and 1.470 at $405 \mathrm{~nm}$ (Michalski et al., 2001; Lopez et al., 2011). Resuspended MFG were diluted in SMUF at pH 6.5 to yield a suspension with a similar fat content to that of the original milk. The diluted MFG solutions or milk were mixed with 35 mM EDTA:NaOH containing 2\% (wt/vol) SDS buffer solution (final $\mathrm{pH} 7.0$ ) in a $1: 1$ ( $\mathrm{vol} / \mathrm{vol}$ ) ratio before loading into the particle size analyzer flow cell. The transparency of the sample cell for both light sources (655 and $405 \mathrm{~nm}$ ) was around 90\%. Measurements were carried out in triplicate. Specific surface area (SSA) of MFG was calculated from the surface area-weighted mean diameter $\left(d_{32}\right)$ : SSA $=6 \varphi / d_{32}$, where $\varphi$ is the volume fraction of milk fat (Huppertz and Kelly, 2006).

\section{Total Lipid Extraction and Quantification}

A Folch extraction (Folch et al., 1957; Hundrieser et al., 1984) was carried out to extract total lipids from milk and MFG fraction samples for subsequent PL extraction and total fat content determination. Samples were mixed with chloroform:methanol $(2: 1, \mathrm{vol} / \mathrm{vol})$ solvent in a 1:8 (vol/vol) ratio for milk or 1:20 (wt/vol) for MFG samples, respectively. The mixtures were washed with 0.2 volumes of $0.9 \%$ (wt/ $\mathrm{vol}$ ) $\mathrm{NaCl}$ solution to increase total lipid recovery. The chloroform-enriched lipid fraction phase was collected and the solvents were dried by rotary evaporation (or by a gentle nitrogen stream when the volume of solvent was not more than $3 \mathrm{~mL}$ ). All extractions were carried out in triplicate. 


\section{Characterization and Quantification of Phospholipids}

The method used for PL extraction and determination was adopted from Avalli and Contarini (2005), with slight modifications. Briefly, PL of MFG were extracted by solid-phase extraction (SPE) from the whole lipid fraction obtained from the Folch extraction. Extracted total lipids were dissolved in chloroform-methanol (2:1, vol/vol) to obtain $400 \mathrm{mg} / \mathrm{mL}$ of lipids for SPE. A fraction of this total lipid solution $(0.5 \mathrm{~mL})$ was applied to silica gel-bonded SPE cartridges (Supelclean LC-SI, 6-mL volume, 1-g sorbent, Supelco, Bellefonte, PA), which were preconditioned with $3 \mathrm{~mL}$ of hexane. Nonpolar lipids were first eluted with $3 \mathrm{~mL}$ of hexane:diethyl ether $(8: 2, \mathrm{vol} / \mathrm{vol})$ and $3 \mathrm{~mL}$ of hexane:diethyl ether $(1: 1, \mathrm{vol} / \mathrm{vol})$ followed by washing the PL from the SPE cartridge with $2 \mathrm{~mL}$ of methanol and $2 \mathrm{~mL}$ of chloroform:methanol:water (3:5:2, vol/ $\mathrm{vol} / \mathrm{vol})$ and collected in SPE vacuum manifolds (12 ports, Sigma-Aldrich). Recovered PL were dried under nitrogen gas and stored at $-80^{\circ} \mathrm{C}$ before analysis by HPLC. The PL extraction was carried out in triplicate.

The PL were separated using an Agilent 1200 HPLC system (Agilent Technologies, Santa Clara, $\mathrm{CA}$ ) and detected with an evaporative light scattering detector (Agilent Technologies). Five major PL-PE, PI, PS, PC, and SM-were separated using an analytical Zorbax Rx-SIL, $4.6 \mathrm{~mm}$ i.d. $\times 250 \mathrm{~mm}$, 5 - $\mu \mathrm{m}$ column (Agilent Technologies) on an HPLC system with a linear binary gradient program $\left[t_{0}\right.$ min: $0 \% \mathrm{~B}, t_{14}: 100 \% \mathrm{~B}, t_{23}: 100 \% \mathrm{~B}, t_{35}: 0 \% \mathrm{~B}, t_{40}: 0 \%$ $\mathrm{B}$; where eluent $\mathrm{A}=$ chloroform:methanol:ammonium hydroxide (80:19.5:0.5, vol/vol/vol) and eluent $\mathrm{B}=$ chloroform:methanol:ammonium hydroxide:water (60:34:0.5:5.5, $\mathrm{vol} / \mathrm{vol} / \mathrm{vol} / \mathrm{vol})]$ at a flow rate of 1.0 $\mathrm{mL} / \mathrm{min}$. Column efficiency before and after PL analysis was tested with meta-xylene and nitrobenzene as analytes and hexane:acetonitrile $(99: 1, \mathrm{vol} / \mathrm{vol})$ as the mobile phase to ensure good performance in PL separation. For PL detected by the evaporative light scattering detector, drift tube temperature was controlled at $50^{\circ} \mathrm{C}$, the pressure of the nebulizer gas (nitrogen) was controlled at $0.31 \pm 0.01 \mathrm{MPa}$, and the signal gain was set to 6 in the Agilent ChemStation software. Identification of PL was done by comparing with the elution times of specific PL standards, and quantitation of PL was calculated from chromatographic peak areas. Standard curves of PL were obtained by injecting a series with known amounts of standards $(5 \mathrm{mg} / \mathrm{mL}$ for $\mathrm{PE}$, $\mathrm{PC}$, and $\mathrm{SM} ; 1 \mathrm{mg} / \mathrm{mL}$ for PI and PS) in gradient injection volumes (from 1 to $10 \mu \mathrm{L}, 5$ levels) in the HPLC. A linear regression was calculated for each standard.

\section{Saponification, Extraction, and Determination of Cholesterol}

The total cholesterol contents of milk and MFG fractions were determined by an enzymatic colorimetric assay on a Roche/Hitachi analysis system using the "cholesterol mode" (CHOL kit, Cobas, Roche/Hitachi, Indianapolis, IN) after cholesterol saponification and extraction. The extraction procedure was carried out (Grossmann et al., 1976; Viturro et al., 2010; Ramalho et al., 2011) with modifications. Briefly, $1.0 \mathrm{~mL}$ of milk sample or $0.1 \mathrm{~g}$ of MFG fraction sample was mixed with $2.4 \mathrm{~mL}$ of $95 \%$ ( $\mathrm{vol} / \mathrm{vol}$ ) ethanol and $1.6 \mathrm{~mL}$ of $50 \%$ (wt/vol) KOH solution. Subsequently, the whole mixture was kept at $70^{\circ} \mathrm{C}$ for 30 min with agitation for saponification to take place. Deionized water $(2 \mathrm{~mL})$ was added into the mixture after saponification for cooling; $2 \mathrm{~mL}$ of $n$-hexane was then added and vortexed for $1 \mathrm{~min}$ for cholesterol extraction. The mixed solution was centrifuged at $2,500 \times g$ for $10 \mathrm{~min}$ for phase separation. The upper cholesterol and $n$-hexane phase was collected and the solvent was evaporated under a gentle nitrogen stream at ambient temperature. The nitrogen-dried cholesterol sample was redissolved in 1 $\mathrm{mL}$ of isopropanol and centrifuged at 21,000 $\times \mathrm{g}$ for 3 min at ambient temperature. The obtained supernatant was stored at $-80^{\circ} \mathrm{C}$ within $24 \mathrm{~h}$ before the final cholesterol determination by enzymatic colorimetric assay. The system was precalibrated with a standard blood serum sample (Roche, Basel, Switzerland) containing a known amount of cholesterol. The loading volumes of cholesterol in samples for colorimetric analysis were all within the assay capacity $(0.03-8 \mathrm{mg} / \mathrm{mL})$.

\section{Estimation of Values of Hydrophilic-Lipophilic Balance of Phospholipids}

The hydrophilic-lipophilic balance (HLB) of MFG phospholipids was calculated using Griffin's method (Griffin, 1954; Ishii and Nii, 2005; Fukuhira et al., 2009), in which the HLB was determined from the ratio of the hydrophilic portion mass to the whole molecular mass. Each type of MFG phospholipid has several sub-species with different FA compositions; therefore, only the major sub-species of each PL [PE (16:0/18:0/18:1/18:2); PI (18:0/18:1); PS (18:0/18:1); PC (16:0/18:1); SM (16:0/23:0/24:0); MacGibbon and Taylor, 2006] were considered to simplify the calculation.

\section{Statistical Analysis}

Significant differences $(P<0.05)$ between samples were determined by one-way ANOVA using Minitab 16 (Minitab Inc., State College, PA). 
Table 1. Quantitation of total polar lipids and cholesterol in raw milk and milk fat globule (MFG) washed fractions on both a total fat weight ( $\mathrm{mg} / \mathrm{g}$ of fat) and specific surface area $\left(\mathrm{mg} / \mathrm{m}^{2}\right.$ of $\mathrm{MFG}$ ) basis ${ }^{1}$

\begin{tabular}{|c|c|c|c|c|}
\hline \multirow[b]{2}{*}{ Sample } & \multicolumn{2}{|c|}{ Total polar lipids } & \multicolumn{2}{|c|}{ Cholesterol } \\
\hline & $\mathrm{mg} / \mathrm{g}$ of fat & $\mathrm{mg} / \mathrm{m}^{2}$ of $\mathrm{MFG}$ & $\mathrm{mg} / \mathrm{g}$ of fat & $\mathrm{mg} / \mathrm{m}^{2}$ of $\mathrm{MFG}$ \\
\hline \multicolumn{5}{|l|}{ M1 } \\
\hline Raw milk & $4.69 \pm 0.34^{\mathrm{b}}$ & $2.76 \pm 0.20^{\mathrm{b}}$ & $3.06 \pm 0.29^{\mathrm{a}}$ & $1.80 \pm 0.17^{\mathrm{bcd}}$ \\
\hline Cream & $2.49 \pm 0.07^{\mathrm{ef}}$ & $1.59 \pm 0.04^{\mathrm{fg}}$ & $2.59 \pm 0.13^{\mathrm{b}}$ & $1.65 \pm 0.09^{\text {bcdef }}$ \\
\hline wash 1 & $2.22 \pm 0.06^{\mathrm{h}}$ & $1.65 \pm 0.05^{\text {cdef }}$ & $2.00 \pm 0.12^{\mathrm{c}}$ & $1.49 \pm 0.09^{\mathrm{f}}$ \\
\hline wash 2 & $2.14 \pm 0.05^{\mathrm{hi}}$ & $1.66 \pm 0.04^{\text {cdef }}$ & $2.03 \pm 0.14^{\mathrm{c}}$ & $1.57 \pm 0.11^{\mathrm{ef}}$ \\
\hline wash 3 & $1.99 \pm 0.11^{\mathrm{i}}$ & $1.70 \pm 0.07^{\text {cde }}$ & $1.85 \pm 0.29^{\mathrm{c}}$ & $1.58 \pm 0.24^{\mathrm{ef}}$ \\
\hline \multicolumn{5}{|l|}{ M2 } \\
\hline Raw milk & $4.78 \pm 0.35^{\mathrm{b}}$ & $2.81 \pm 0.20^{\mathrm{b}}$ & $3.10 \pm 0.30^{\mathrm{a}}$ & $1.82 \pm 0.17^{\mathrm{b}}$ \\
\hline Cream & $2.54 \pm 0.15^{\mathrm{e}}$ & $1.62 \pm 0.09^{\mathrm{efg}}$ & $2.70 \pm 0.47^{\mathrm{b}}$ & $1.72 \pm 0.30^{\text {bcde }}$ \\
\hline wash 1 & $2.41 \pm 0.20^{\mathrm{fg}}$ & $1.55 \pm 0.13^{\mathrm{g}}$ & $2.71 \pm 0.22^{\mathrm{b}}$ & $1.75 \pm 0.14^{\text {bcde }}$ \\
\hline \multicolumn{5}{|l|}{ M3 } \\
\hline Raw milk & $5.00 \pm 0.45^{\mathrm{a}}$ & $2.94 \pm 0.27^{\mathrm{a}}$ & $3.08 \pm 0.26^{\mathrm{a}}$ & $1.81 \pm 0.15^{\mathrm{bc}}$ \\
\hline Cream & $2.89 \pm 0.11^{\mathrm{c}}$ & $1.76 \pm 0.07^{\mathrm{c}}$ & $2.64 \pm 0.22^{\mathrm{b}}$ & $1.61 \pm 0.14^{\mathrm{def}}$ \\
\hline wash 1 & $2.72 \pm 0.16^{\mathrm{cd}}$ & $1.73 \pm 0.10^{\mathrm{cd}}$ & $2.44 \pm 0.19^{\mathrm{b}}$ & $1.55 \pm 0.12^{\mathrm{ef}}$ \\
\hline wash 2 & $2.62 \pm 0.08^{\mathrm{de}}$ & $1.62 \pm 0.05^{\mathrm{defg}}$ & $2.61 \pm 0.22^{\mathrm{b}}$ & $1.61 \pm 0.14^{\text {cdef }}$ \\
\hline wash 3 & $2.26 \pm 0.10^{\mathrm{gh}}$ & $1.56 \pm 0.07^{\mathrm{fg}}$ & $3.35 \pm 0.11^{\mathrm{a}}$ & $2.31 \pm 0.07^{\mathrm{a}}$ \\
\hline
\end{tabular}

${ }^{\mathrm{a}-\mathrm{i}}$ Data within the same column that do not share a letter are different $(P<0.05)$.

${ }^{1}$ Results are means $\pm \mathrm{SD} ; \mathrm{n}=9$ for raw milk and washed fractions used in $\mathrm{M} 1$ and $\mathrm{M} 3 ; \mathrm{n}=18$ for raw milk and washed fraction used in M2.

\section{RESULTS AND DISCUSSION}

\section{Quantification of Total PL and Cholesterol}

Approximately 60 to $65 \%$ of PL are associated with the MFGM on the globule surface and the rest presents in the aqueous phase of raw milk (MacGibbon and Taylor, 2006). Desorption of PL may occur by mechanical disturbance of milk during transportation and processing (Walstra et al., 2006). We have assumed that all PL and cholesterol were associated with the MFGM (regardless of the size of MFG) in our unprocessed milk samples. This assumption does not affect subsequent calculations of relative changes in these components during processing.

The total amounts of PL and cholesterol in the MFGM were calculated as a proportion of total fat $(\mathrm{mg} / \mathrm{g})$ to quantify relative changes of each compound, and also calculated on an MFG surface area basis from SSA (mg/ $\mathrm{m}^{2}$ ) values to characterize the surface changes (Table 1). Our results for PL content in milk are comparable to reported results, $5.1 \pm 1.7 \mathrm{mg} / \mathrm{g}$ of lipid (Patton and Huston, 1986), $2.5 \pm 1.4 \mathrm{mg} / \mathrm{g}$ of lipid (Lopez et al., 2008), $6.3 \pm 0.5 \mathrm{mg} / \mathrm{g}$ of lipid (Lopez et al., 2011), and $9.8 \pm 0.0 \mathrm{mg} / \mathrm{g}$ of lipid (Rombaut et al., 2006), although different MFG isolation methods were applied in each study. In the current study, the PL content of milk samples used for the M3 process $(\sim 5.00 \mathrm{mg} / \mathrm{g}$ of fat) was different $(P<0.001)$ than that of milk for the M1 process $(\sim 4.69 \mathrm{mg} / \mathrm{g}$ of fat), although the samples were sourced from same cow (mid-lactation stage), and the feed of the cow was not changed over the 6-d milking period. Despite this, we found no significant difference $(P<0.001)$ in cholesterol content between milk samples before the M1 and M3 processes. A diet enriched in PUFA induces a higher yield of PL (Lopez et al., 2008); however, the feed in the current study was controlled, suggesting that other environmental factors may affect the yield of PL. The yield of cholesterol was more consistent across raw milk samples (Table 1) compared with PL (detailed data not shown), suggesting that the yield or secretion of cholesterol is less environmentally dependent than that of PL.

Total PL content of MFG decreased significantly ( $P$ $<0.001)$ after the initial centrifugation of milk for all 3 processes, and the most intensive process (M3) yielded the greatest amount of PL on a weight and surface area basis (Table 1). Although depletion of MFGM material occurs during centrifugation (Walstra et al., 2006), more intensive initial centrifugation of milk can collect a greater volume of smaller MFG into the cream phase. Smaller lipid structures, including fat globules, have more PL content (based on both fat weight and SSA) due to a smaller proportion (and in some cases, absence) of the triglyceride inner core of small vesiclelike structures $<2 \mu \mathrm{m}$, and with a higher total surface area for smaller particles (Gallier et al., 2011; Lopez et al., 2011). Consequently, greater recovery of PL is expected when more of the smaller lipid structures are collected by centrifugation. Generally, a loss of total PL after methods M1, M2, and M3 was found when PL content was calculated on a total fat basis (Table 1). Surprisingly, when the SSA of MFG was used as the basis for the calculation of PL content in the M1 process, 
relative enrichment rather than loss of PL content was found from the cream to the third wash, but a significant loss of PL was found between the cream and the third wash in the M3 process (Table 1). The apparent relative enrichment of PL on a surface area-weighted basis was due to less depletion after the mild washing process with a concomitant increase in the surface areaweighted mean diameter of MFG $\left(d_{32}\right)$, and therefore a decrease in SSA. These results are in good agreement with Zheng et al. (2013), where it was reported that the milder M1 process did not induce destructive damage to the PL outer bilayer of the MFGM, whereas the M3 process exposed the inner protein layer of the MFGM after the second wash, which was deemed to be destructive to the outer bilayer. The M2 and M3 washing processes are recommended when a high total yield of PL is required; however, the M1 process is recommended if damage to the MFG surface should be minimized.

For validation of the cholesterol assay, a standard addition method was carried out by spiking the MFG fractions with pure cholesterol; the calculated recovery of cholesterol from the fractions was $99.8 \pm 4.6 \%$. The cholesterol values (Table 1) compare well with the reported value of $3.3 \mathrm{mg} / \mathrm{g}$ of fat in raw milk (MacGibbon and Taylor, 2006). We detected a significant decrease $(P<0.001)$ in cholesterol content from raw milk samples to the first washing step for all 3 methods on both a weight and surface area basis, with the exception of the surface area-weighted data for the M2 process (Table 1). The extent of cholesterol depletion from the raw milk samples was less than the depletion of PL, suggesting that cholesterol is a relatively tightly bound component within the MFGM. This supports the structural model systems of the MFGM in which cholesterol is reported to interact with SM to form rigid $L_{o}$ domains (lipid rafts) in the MFGM bilayer (Lopez et al., 2010, 2011). Surprisingly, an apparent relative enrichment of cholesterol on both a surface area and weight basis was found from cream to the M3-3 wash sample, in contrast to the depletion of total PL. This suggests that cholesterol is not desorbed together with PL as integral MFGM fragments after the intensive centrifugation treatment. Lee et al. (1999) also reported that increased centrifugal force may decrease the depletion of cholesterol from MFG surfaces.

These observations suggest the following structural changes in the MFGM during washing. The PL in the $L_{d}$ domain of the outer leaflet of the MFGM PL bilayer (Gallier et al., 2010b; Lopez et al., 2010, 2011) that are not associated with cholesterol are released from the surface of MFG. The MFGM bilayer may rupture and the rigid $L_{o}$ domains containing cholesterol then become exposed to the aqueous phase and repartition from the outer bilayer to the primary inner monolayer of the MFGM. The cholesterol content was enriched when the outer bilayers of MFG were damaged; a process of cholesterol partitioning from MFGM to the core of the MFG was discussed by Evers (2004).

The relative increase in cholesterol content on a surface area-weighted basis on the MFG surfaces can be considered as another indicator for assessing damage to the MFGM. If apparent relative enrichment of cholesterol on MFG surfaces is observed during mechanical treatment, then destructive damage to the outer bilayer of MFGM is likely. As a consequence, this suggests that the M1 and M2 washing processes do not induce destructive damage to the outer bilayer of the MFGM, whereas the M3 process damages the bilayer during the third washing step. This is in good agreement with confocal laser scanning microscopy images, where damage to the PL bilayer and exposure of the inner monolayer containing membrane proteins after the M3 washing process was observed (Zheng et al., 2013).

\section{Relative Depletion or Enrichment of Individual Phospholipids}

The quantification of individual PL by linear regression gave a high coefficient of determination $\left(\mathrm{R}^{2}>\right.$ 0.99). A standard addition procedure was carried out to measure PE and SM recovery, yielding $99.0 \pm 2.3 \%$ for $\mathrm{PE}$ and $96.7 \pm 0.2 \%$ for SM. The use of PL standards derived from milk gave a higher recovery compared with previous work (Avalli and Contarini, 2005), where standards derived from bovine brain were used. Typical chromatograms from samples of M3-cream and M3-3 washed fractions show the regions where the 5 major PL were eluted between 10 and 20 min (Figure 1). Large amounts of neutral lipids were eluted from the analytical HPLC column within the first $5 \mathrm{~min}$, and no noticeable peaks were shown after $20 \mathrm{~min}$ (results not shown). The phospholipids PE, PC, and SM accounted for most of the total PL content, and PI and PS were minor components. Four distinguishable sub-peaks of $\mathrm{PC}\left(\mathrm{PC}_{2}>\mathrm{PC}_{1}>\mathrm{PC}_{3}>\mathrm{PC}_{4}\right)$ and 3 sub-peaks of $\mathrm{SM}$ $\left(\mathrm{SM}_{2}>\mathrm{SM}_{3}>\mathrm{SM}_{1}\right)$, ordered in terms of peak area, were found (Figure 1), as observed by others (Avalli and Contarini, 2005; Rombaut et al., 2005; Lopez et al., 2011). The peak profile of PC changed after 3 washes in the M3 process (Figure $1 \mathrm{~A}$ and B). Although the detailed structural organization of these 4 species of $\mathrm{PC}$ is unknown, this observation implies an asymmetric distribution of the different PC isomers in the MFGM. One of the PC isomers in milk, 1,2-dipalmitoyl-snglycero-3-phosphocholine (DPPC), is believed to form tightly packed segregated lipid domains at ambient temperature in an artificial giant unilamellar vesicle phospholipid bilayer system (Zheng et al., 2014). 


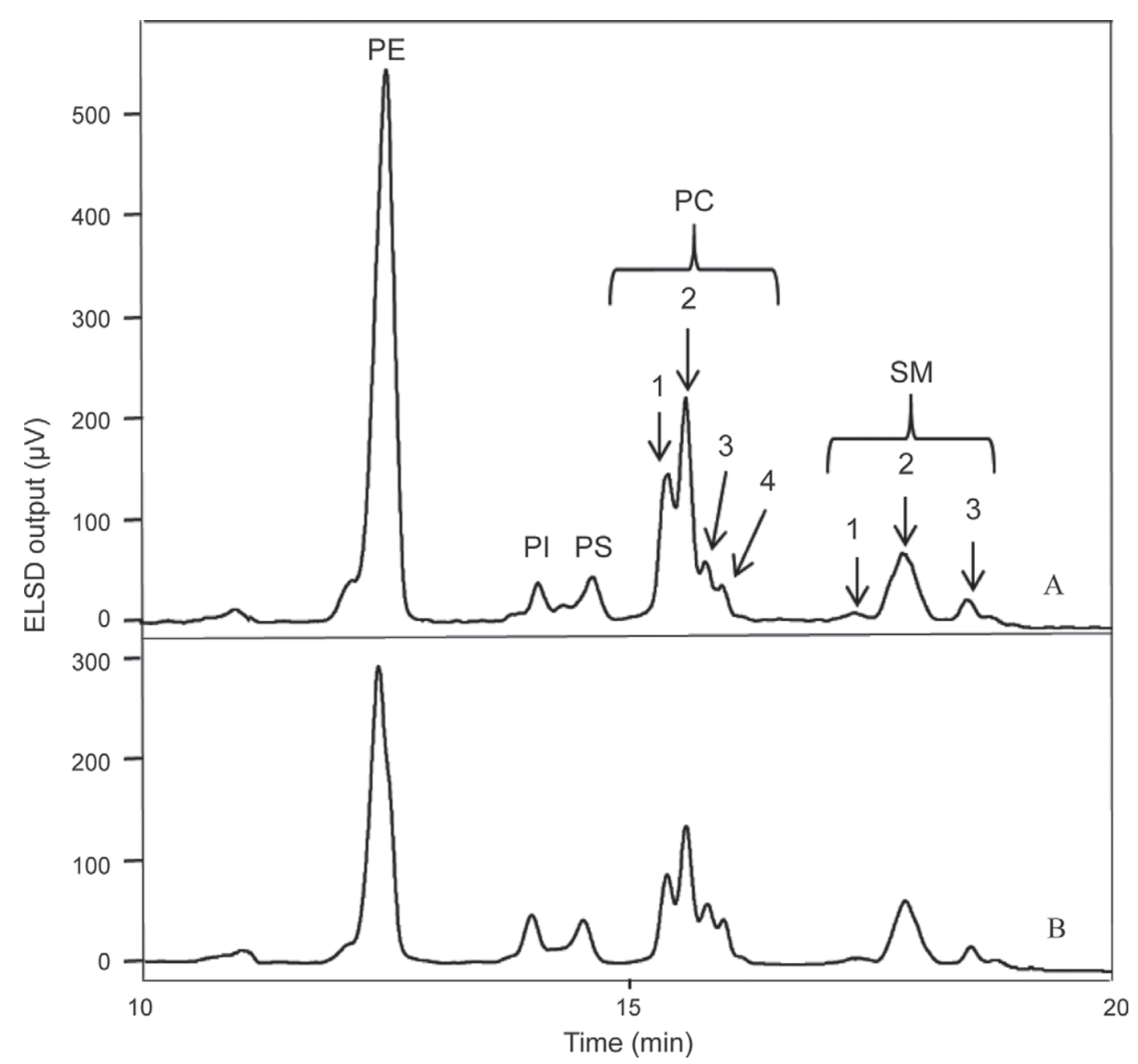

Figure 1. High-performance liquid chromatography chromatograms with evaporative light-scattering detection (ELSD) of lipids on the milk fat globule membrane for (A) M3-cream and (B) M3-3 wash. PE = phosphatidylethanolamine, $\mathrm{PC}=$ phosphatidylcholine, PI $=$ phosphatidylinositol, PS = phosphatidylserine, SM = sphingomyelin. Marked numbers are sub-peaks for PC and SM.

The proportions of 5 major PL in the MFGM were calculated on a surface area-weighted basis (Table 2). Generally, the initial centrifugation step for producing cream changed the proportions of individual PL on the surface for all 3 washing processes, likely due to (1) desorption of MFGM material under centrifugal forces, (2) the removal of free nonadsorbed PL (or MFGM fragments) from the aqueous phase of milk and cream, or (3) changes in the SSA of MFG as a consequence of a shift in the size distribution. Of these 3 mechanisms, the first is the one more likely to damage the MFG surface and thus show potential to reveal any structural changes that occur during processing.

Comparisons of apparent relative depletion and enrichment of individual PL on MFG surfaces was carried out with the initial isolated creams and the washed MFG suspensions in the M1 and M3 processes. The data from the original milk were excluded from the comparison to eliminate the confounding factor of free nonadsorbed PL, MFGM fragments, and MFGM vesicles. During the M1 washing process, when damage to the MFGM did not occur, relative desorption of $\mathrm{PE}$ and $\mathrm{PC}$ from the surfaces of MFG was found from the cream to the M1-3 wash (Table 2), whereas the amount of SM increased after the M1-3 wash from the cream. A similar trend for these 3 phospholipids was also found for the M3 method. The proportion of PE increased from M3-2 to M3-3; therefore, it is reasonable to deduce that some of the $\mathrm{PE}$ were repartitioning from the outer bilayer into the inner monolayer (similar to the repartitioning of cholesterol) during the third wash of the M3 process when the outer bilayer was damaged due to its peripheral location in the MFGM. Both PC and $\mathrm{PE}$ can be considered as loosely PL as they were partially depleted from the surfaces of MFG in the M1 and M3 processes. These phenomena must relate to the 
Table 2. Percentage composition of specific phospholipids on milk fat globule surfaces calculated on a specific surface area (SSA) basis ${ }^{1}$

\begin{tabular}{|c|c|c|c|c|c|}
\hline \multirow[b]{2}{*}{$\%$ SSA basis } & \multicolumn{5}{|c|}{ Phospholipid $^{2}$} \\
\hline & $\mathrm{PE}$ & PI & PS & $\mathrm{PC}$ & SM \\
\hline \multicolumn{6}{|l|}{ M1 } \\
\hline Raw milk & $27.15 \pm 0.35^{\mathrm{fg}}$ & $5.84 \pm 0.27^{\mathrm{g}}$ & $14.12 \pm 1.00^{\mathrm{efg}}$ & $26.94 \pm 0.64^{\mathrm{a}}$ & $25.62 \pm 0.62^{\mathrm{a}}$ \\
\hline Cream & $31.28 \pm 0.97^{\mathrm{ab}}$ & $8.55 \pm 0.60^{\mathrm{ab}}$ & $12.19 \pm 0.42^{\mathrm{ef}}$ & $24.25 \pm 0.99^{\mathrm{cd}}$ & $24.06 \pm 0.89^{\mathrm{c}}$ \\
\hline 1 wash & $31.20 \pm 0.86^{\mathrm{ab}}$ & $8.85 \pm 0.27^{\mathrm{a}}$ & $11.65 \pm 0.49^{\mathrm{de}}$ & $23.64 \pm 0.76^{\mathrm{de}}$ & $24.61 \pm 0.52^{\mathrm{bc}}$ \\
\hline 2 wash & $29.97 \pm 0.96^{\text {cde }}$ & $8.33 \pm 0.33^{\mathrm{bcd}}$ & $13.60 \pm 0.83^{\mathrm{g}}$ & $23.48 \pm 1.04^{\mathrm{de}}$ & $24.56 \pm 0.53^{\mathrm{bc}}$ \\
\hline 3 wash & $29.91 \pm 1.04^{\mathrm{de}}$ & $8.43 \pm 0.28^{\mathrm{bc}}$ & $13.81 \pm 0.79^{\mathrm{fg}}$ & $22.82 \pm 0.78^{\mathrm{ef}}$ & $25.16 \pm 0.49^{\mathrm{ab}}$ \\
\hline \multicolumn{6}{|l|}{ M2 } \\
\hline Raw milk & $27.30 \pm 0.64^{\mathrm{f}}$ & $5.77 \pm 0.28^{\mathrm{g}}$ & $14.55 \pm 1.00^{\mathrm{ef}}$ & $27.35 \pm 0.89^{\mathrm{a}}$ & $25.59 \pm 0.79^{\mathrm{a}}$ \\
\hline Cream & $26.50 \pm 0.71^{\mathrm{g}}$ & $8.16 \pm 0.48^{\mathrm{cd}}$ & $16.15 \pm 1.18^{\mathrm{bc}}$ & $25.22 \pm 1.41^{\mathrm{b}}$ & $24.21 \pm 0.88^{\mathrm{c}}$ \\
\hline 1 wash & $26.45 \pm 0.80^{\mathrm{g}}$ & $8.25 \pm 0.45^{\mathrm{cd}}$ & $15.67 \pm 1.47^{\mathrm{cd}}$ & $25.19 \pm 1.42^{\mathrm{b}}$ & $24.23 \pm 1.07^{\mathrm{c}}$ \\
\hline \multicolumn{6}{|l|}{ M3 } \\
\hline Raw milk & $27.35 \pm 0.58^{\mathrm{f}}$ & $5.73 \pm 0.29^{\mathrm{g}}$ & $15.02 \pm 0.99^{\mathrm{de}}$ & $27.40 \pm 1.15^{\mathrm{a}}$ & $25.45 \pm 1.43^{\mathrm{a}}$ \\
\hline Cream & $32.02 \pm 2.95^{\mathrm{a}}$ & $7.34 \pm 0.92^{\mathrm{f}}$ & $16.75 \pm 1.36^{\mathrm{ab}}$ & $25.87 \pm 1.80^{\mathrm{b}}$ & $20.93 \pm 0.91^{\mathrm{ef}}$ \\
\hline 1 wash & $30.73 \pm 1.25^{\mathrm{bc}}$ & $7.73 \pm 0.64^{\mathrm{ef}}$ & $16.98 \pm 0.62^{\mathrm{a}}$ & $25.00 \pm 1.15^{\mathrm{bc}}$ & $20.65 \pm 0.87^{\mathrm{f}}$ \\
\hline 2 wash & $29.31 \pm 0.62^{\mathrm{e}}$ & $7.46 \pm 1.06^{\mathrm{f}}$ & $16.33 \pm 1.61^{\mathrm{abc}}$ & $23.91 \pm 0.46^{\mathrm{d}}$ & $21.46 \pm 0.61^{\mathrm{e}}$ \\
\hline 3 wash & $30.52 \pm 0.87^{\mathrm{bcd}}$ & $8.00 \pm 0.28^{\mathrm{de}}$ & $16.78 \pm 0.48^{\mathrm{ab}}$ & $22.46 \pm 1.01^{\mathrm{f}}$ & $22.89 \pm 1.82^{\mathrm{d}}$ \\
\hline
\end{tabular}

structural properties and specific locations of PL in the MFGM.

Although the total PL content of raw milk samples varied across different milking days (data not shown), the proportions of individual PL were not significantly different $(P<0.001$; Table 2$)$. The proportions of each major PL in milk (Table 2) were comparable with previously published work: $\mathrm{PE} \sim 23.2 \%$, PI $\sim 8.1 \%$, PS $16.1 \%, \mathrm{PC} \sim 26.6 \%$, and $\mathrm{SM} \sim 26.0 \%$ (wt/wt; Lopez et al., 2011), but different from that reported by Rombaut et al. (2005) where the PE content was higher than $40 \%$ and PC and SM were lower than 20\%. MacGibbon and Taylor (2006) noted that the measured proportions of individual PL depend upon the analytical methods used, breed of the cow, diet, and stage of lactation. The extraction method of PL from milk is also a crucial factor (Avalli and Contarini, 2005; Gallier et al., 2010a).

\section{Updated Model of PL Organization in the MFGM}

An updated model system of lipid organization in the MFGM is proposed from observations of depletion of PL from MFG surfaces and integrating features from previous models (Michalski et al., 2002; Lopez et al., 2011). In the current proposed schematic model (Figure 2 ), the outer bilayer is separated into ordered domains (gel phase: black-filled head groups of SM without cholesterol; $L_{o}$ : black-filled PC and SM with cholesterol) and liquid-disordered domains ( $L_{d}$, gray-filled head groups). Lissamine rhodamine B-labeled phospholipid fluorescent dye was found to be segregated from the gel domains induced by this dye, and was located in the DPPC liquid crystalline domains at intermediate temperature on a nonsupported artificial phospholipid bilayer system (Bagatolli and Gratton, 2000). Moreover, recent work showed that a gel phase composed of DPPC without the inclusion of cholesterol and SM may contribute to lipid domain formation in a giant unilamellar vesicle system containing only DPPC and 1,2-dioleoyl-sn-glycero-3-phosphoethanolamine (Zheng et al., 2014). These results suggest that lipid segregation in the MFGM, as previously characterized using confocal microscopy after fluorescent staining with lissamine rhodamine B-labeled 1,2-dioleoyl-sn-glycero3-phosphoethanolamine (Gallier et al., 2010b; Lopez et al., 2010), is not only represented by $L_{o}$ domains as shown in previous schematic model systems (Lopez et al., 2010, 2011), but also by gel domains (Figure 2). It has been reported that SM displays a gel-liquid transition around physiological temperatures (Barenholz and Thompson, 1980; Contreras et al., 2004), which rationalizes the co-existence of gel and liquid states of SM (Figure 2).

The glycerolphospholipids phosphosphingolipids and cholesterol are asymmetrically distributed in the trilayer system (Figure 2). The proposed schematic model system contains $19 \mathrm{PL}$ and 3 cholesterol molecules in simulating the lipid composition of the native MFGM. In the proposed model, PE, PC, and SM account for $26 \%$ each, and PS, PI, and cholesterol account for 16 , 11 , and $16 \%(\mathrm{~mol} / \mathrm{mol})$, respectively, of total phospholipid and cholesterol molecules. This lipid composition 


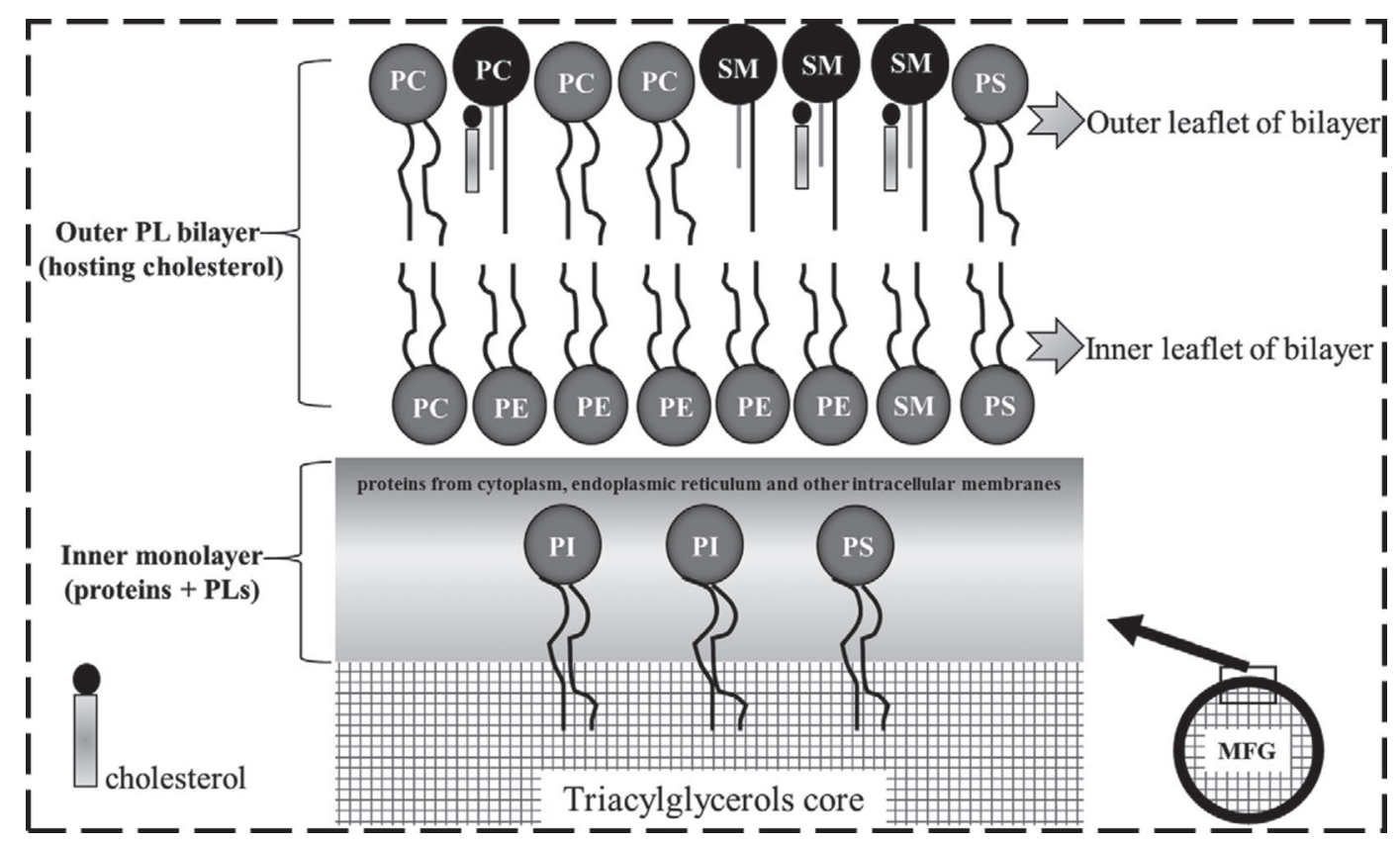

Figure 2. Schematic model of lipid organization of the bovine milk fat globule (MFG) membrane showing the outer bilayer and inner monolayer. On the outer leaflet of the bilayer, liquid-ordered regions are shown as phospholipids with headgroups of a darker shade; the gray headgroups show the liquid-disordered regions. $\mathrm{PE}=$ phosphatidylethanolamine, $\mathrm{PC}=$ phosphatidylcholine, $\mathrm{PI}=$ phosphatidylinositol, $\mathrm{PS}=$ phosphatidylserine, $\mathrm{SM}=$ sphingomyelin.

in the model is comparable with the estimated lipid composition of the native MFGM from previously published results (Huppertz and Kelly, 2006; Lopez et al., 2011 ) in which $\mathrm{PE} \sim 23 \%, \mathrm{PC} \sim 26 \%, \mathrm{SM} \sim 26 \%$, PS $\sim 16 \%$, PI $\sim 11 \%$, and cholesterol $\sim 16 \%(\mathrm{~mol} / \mathrm{mol})$.

The HLB values of individual PL decreased in the order $\mathrm{PI}>\mathrm{PS}>\mathrm{SM}>\mathrm{PC}>\mathrm{PE}$ (Table 3 ); however, the closeness of values for the last 4 phospholipids in the list may not have significant structural implications. This additional information further supports the proposed model (Figure 2) as follows: PI and PS form part of the inner monolayer as they are relatively resistant to being removed after washing the MFG suspensions (from raw cream to final wash; Table 2). The PI molecule has the highest HLB value (Table 3) and is therefore more likely to stabilize an oil-in-water emulsion, such as the inner triacylglyceride core, and be located in the inner monolayer. The relatively smaller differences in HLB values of the remaining phospholipids do not allow definitive conclusions to be drawn about locations within the MFGM. The PS may be evenly distributed in both the outer bilayer and inner primary monolayer as we observed no apparent trend in enrichment during the M3 washing processes (Table 2) when the outer bilayer was (partially) washed away, as shown in confocal micrograph images (Zheng et al., 2013). Previous reports have shown that PE, PI, and PS are located within the inner layers of the MFGM, whereas PC and SM are located on the outer surface (Deeth, 1997; Rombaut et al., 2006); however, there is uncertainty about the detailed distribution of PL in the inner layers of the MFGM (the primary PL monolayer covering the triacylglyceride core and the inner half leaflet of the outer bilayer).

The critical packing parameter (which determines the preferred association structure of molecular shape dependent on the effective volume of $\mathrm{FA}$, length of FA, and head group area) of PE is $>1$, and it has an inverted truncated cone critical packing shape, which makes it suitable to have an inverted packing structure (Israelachvili, 2010) in the inner leaflet of bilayer of the interface region between the PL outer leaflet of the bilayer and the PL inner monolayer (Figure 2). This specific location of $\mathrm{PE}$ is supported by the relative enrichment of PE in the MFGM from the second to the third wash in the M3 process (Table 2). Moreover, evidence has been provided using a giant unilamellar vesicle system to show that $\mathrm{PE}$ is located in the inner leaflet of the phospholipid bilayer (Zheng et al., 2014).

During the final wash of the M3 process, cholesterol was relatively enriched in the MFG, possibly because of the partitioning of cholesterol from the outer bilayer to the primary monolayer. A similar hypothesis could be made for the repartitioning of PE when the bilayer was damaged after the final wash in the M3 process to explain the small relative enrichment from M3-2 to M3-3 
Table 3. Estimation of hydrophilic-lipophilic balance (HLB) value of phospholipids

\begin{tabular}{|c|c|c|c|c|c|}
\hline \multirow[b]{2}{*}{ Item } & \multicolumn{5}{|c|}{ Phospholipid $^{1}$} \\
\hline & $\mathrm{PE}$ & PI & PS & $\mathrm{PC}$ & $\mathrm{SM}$ \\
\hline Fatty acids ${ }^{2}$ & $16: 0 / 18: 0 / 18: 1 / 18: 2$ & $18: 0 / 18: 1$ & $18: 0 / 18: 1$ & $16: 0 / 18: 1$ & $16: 0 / 23: 0 / 24: 0$ \\
\hline Estimated HLB & 2.6 & 8.4 & 3.9 & 3.6 & 3.7 \\
\hline
\end{tabular}

wash (Table 2). This small degree of relative enrichment of PE may also be due to the proposed specific location in the native MFGM, as shown in Figure 2.

The majority of PC is likely present in the external part of the PL bilayer as PC was partially desorbed in the M1 and M3 washing processes (Table 2), due to a loose arrangement of PL in the outer layer of the MFGM (Rombaut et al., 2006). The proportions of PC and SM decreased after initial centrifugation for cream isolation in all 3 processes (more so for SM in the M3 process), whereas other PL increased in relative proportion, with the exception being PS in the M1 process, which remained the same (Table 2). This suggests that $\mathrm{PC}$ and SM are both present in the outer layer of the MFGM (Deeth, 1997; Danthine et al., 2000; Rombaut et al., 2006). Moreover, cholesterol content did not change significantly from M1-raw to M1-cream (surface area-weighted basis, Table 1), which, together with the significant desorption of SM in the paired samples (Table 2), suggests that not all SM molecules interact with cholesterol (Figure 2).

The outer leaflet of PL bilayers of biological membranes, such as the plasma membranes of human and animal cells, is enriched with zwitterionic PC and SM (Op den Kamp, 1979; McMullen et al., 2004). A similar observation was discussed by Rombaut and coworkers (2006), who reported that PE, PI, and PS were negatively correlated with PC and SM during processinduced depletion, and who also noted a high SM content in skim milk. Therefore, in the current results, the increase in the proportion of SM from cream to 3-times washed MFG and the decrease from raw milk to cream for the M1 and M3 processes (Table 2) suggests that some of the SM is held rigidly in the MFGM structure and some held more loosely, as shown in Figure 2. The rigidly held SM in the MFGM can be considered as indirect evidence for the existence of SM-enriched lipid $L_{o}$ domains, which have been observed by confocal laser scanning microscopy after fluorescent staining (Gallier et al., 2010b; Lopez et al., 2010).

The formation of these $L_{o}$ domains is regulated by cholesterol and they are specifically formed by interac- tion with SM (de Almeida et al., 2009; Lopez et al., 2010). Cholesterol interacts not only with SM but also with other glycerolphospholipids in biological membranes, although SM has a greater affinity for cholesterol than for other PL, such as PC (McMullen et al., 2004). Therefore, some of the cholesterol in the MFGM might interact with other glycerolphospholipids (such as PC), locate within the $L_{o}$ domain, and protrude from the $L_{d}$ domain, as shown in the proposed model system of the MFGM (Figure 2). The existence of an ordered domain from binary mixtures of cholesterol and DPPC (one of the major species of milk PC) was characterized by differential scanning calorimetry and nuclear magnetic resonance spectroscopy (Vist and Davis, 1990; McMullen et al., 2004). The laterally protruding $L_{o}$ domain, containing specific sub-species of PC (longer and saturated acyl chains) and SM in the MFGM (Figure 2 ), can be explained by the higher trans/gauche ratio in the $L_{o}$ domains, causing a thicker structure than for the $L_{d}$ domain (Simons and Vaz, 2004). Results from atomic force microscopy have shown a thicker structure of the $L_{o}$ domain in monolayer model systems formed from milk-derived PL (Gallier et al., 2010c). The gel phase is reported to be thicker than the fluid phase on a supported PL bilayer (Giocondi et al., 2004), which supports the notion of protrusion of $L_{o}$ domains (Figure $2)$.

\section{CONCLUSIONS}

The present study measured changes in relative proportions of the major MFGM polar lipid components and cholesterol on the surfaces of native MFG after centrifugal washing processes with different degrees of stringency. Consequently, we revealed the ease with which 5 major MFGM polar lipids on native MFG surfaces could be removed by washing. The proposed mechanism of repartitioning cholesterol between the MFGM outer bilayer and primary inner monolayer was described based on the relative enrichment of cholesterol after an intensive washing procedure that caused destructive damage to the outer bilayer. The general 
location of major polar lipids in the trilayer structure was deduced from relative depletion and enrichment of components after washing. Evidence was provided that sphingomyelin is located in both the ordered (gel and liquid-ordered) and liquid-disordered regions of the MFGM, and not necessarily in association with cholesterol. This study updates the current understanding of lipid organization in the MFGM, which will have important implications for MFGM extraction and purification as a dairy ingredient and greater understanding of its physiological functions.

\section{ACKNOWLEDGMENTS}

The authors acknowledge the Earle Food Research Fund from the Riddet Institute (Palmerston North, New Zealand). We also thank Ian Ross and Nerida Downes (Department of Food Science, University of Otago, New Zealand) for assistance with HPLC analysis, and Ashley Duncan and Michelle Harper (Department of Human Nutrition, University of Otago, New Zealand) for discussions on the development of a method for measuring the cholesterol content of MFG.

\section{REFERENCES}

Argov, N., D. G. Lemay, and J. B. German. 2008. Milk fat globule structure and function: Nanoscience comes to milk production. Trends Food Sci. Technol. 19:617-623.

Avalli, A., and G. Contarini. 2005. Determination of phospholipids in dairy products by SPE/HPLC/ELSD. J. Chromatogr. A 1071:185-190.

Bagatolli, L. A., and E. Gratton. 2000. Two photon fluorescence microscopy of coexisting lipid domains in giant unilamellar vesicles of binary phospholipid mixtures. Biophys. J. 78:290-305.

Barenholz, Y., and T. E. Thompson. 1980. Sphingomyelins in bilayers and biological membranes. Biochim. Biophys. Acta Rev. Biomembr. 604:129-158.

Contreras, F. X., J. Sot, M. B. Ruiz-Argüello, A. Alonso, and F. M. Goñi. 2004. Cholesterol modulation of sphingomyelinase activity at physiological temperatures. Chem. Phys. Lipids 130:127-134.

Danthine, S., C. Blecker, M. Paquot, N. Innocente, and C. Deroanne. 2000. Progress in milk fat globule membrane research: A review. Lait 80:209-222.

de Almeida, R. F. M., L. M. S. Loura, and M. Prieto. 2009. Membrane lipid domains and rafts: Current applications of fluorescence lifetime spectroscopy and imaging. Chem. Phys. Lipids 157:61-77.

Deeth, H. C. 1997. The role of phospholipids in the stability of milk fat globules. Aust. J. Dairy Technol. 52:44-46.

Dewettinck, K., R. Rombaut, N. Thienpont, T. Le, K. Messens, and J. Vancamp. 2008. Nutritional and technological aspects of milk fat globule membrane material. Int. Dairy J. 18:436-457.

Evers, J. M. 2004. The milkfat globule membrane-Compositional and structural changes post secretion by the mammary secretory cell. Int. Dairy J. 14:661-674.

Folch, J., M. Lees, and G. H. S. Stanley. 1957. A simple method for the isolation and purification of total lipids from animal tissues. J. Biol. Chem. 226:497-509.

Fukuhira, Y., H. Yabu, K. Ijiro, and M. Shimomura. 2009. Interfacial tension governs the formation of self-organized honeycomb-patterned polymer films. Soft Matter 5:2037-2041.
Gallier, S., K. C. Gordon, R. Jiménez-Flores, and D. W. Everett. 2011. Composition of bovine milk fat globules by confocal Raman microscopy. Int. Dairy J. 21:402-412.

Gallier, S., D. Gragson, C. Cabral, R. Jiménez-Flores, and D. W. Everett. 2010a. Composition and fatty acid distribution of bovine milk phospholipids from processed milk products. J. Agric. Food Chem. 58:10503-10511.

Gallier, S., D. Gragson, R. Jiménez-Flores, and D. W. Everett. 2010b. Using confocal laser scanning microscopy to probe the milk fat globule membrane and associated proteins. J. Agric. Food Chem. 58:4250-4257.

Gallier, S., D. Gragson, R. Jiménez-Flores, and D. W. Everett. 2010c. Surface characterization of bovine milk phospholipid monolayers by Langmuir isotherms and microscopic techniques. J. Agric. Food Chem. 58:12275-12285.

Giocondi, M.-C., P. E. Milhiet, P. Dosset, and C. L. Grimellec. 2004. Use of cyclodextrin for AFM monitoring of model raft formation. Biophys. J. 86:861-869.

Griffin, W. C. 1954. Calculation of HLB values of non-ionic surfactants. J. Soc. Cosmet. Chem. 5:249-256.

Grossmann, A., H. Timmen, and H. Klostermeyer. 1976. Enzymatic estimation of cholesterol in milk-fat-Alternative to methods in current use. Milchwissenschaft 31:721-724.

Huang, T. C., and A. Kuksis. 1967. A comparative study of the lipids of chylomicron membrane and fat core and of the lymph serum of dogs. Lipids 2:443-452.

Hundrieser, K. E., R. M. Clark, R. G. Jensen, and A. M. Ferris. 1984. A comparison of methods for determination of total lipids in human milk. Nutr. Res. 4:21-26.

Huppertz, T., and A. L. Kelly. 2006. Physical chemistry of milk fat globules. Pages 173-212 in Advanced Dairy Chemistry. Vol. 2. Lipids. P. F. Fox and P. L. H. McSweeney, ed. Springer, New York, NY.

Ishii, F., and T. Nii. 2005. Properties of various phospholipid mixtures as emulsifiers or dispersing agents in nanoparticle drug carrier preparations. Colloids Surf. B Biointerfaces 41:257-262.

Israelachvili, J. N. 2010. Soft and biological structures. Pages 535-576 in Intermolecular and Surface Forces. 3rd ed. Elsevier Science, Burlington, MA.

Jenness, R., and J. Koops. 1962. Preparation and properties of a salt solution which simulates milk ultrafiltrate. Neth. Milk Dairy J. $16: 153-164$.

Keenan, T. W., and I. H. Mather. 2006. Intracellular origin of milk fat globules and the nature of the milk fat globule membrane. Pages 137-171 in Advanced Dairy Chemistry. Vol. 2: Lipids. P. F. Fox and P. L. H. McSweeney, ed. Springer, New York, NY.

Le, T. T., J. Van Camp, R. Rombaut, F. van Leeckwyck, and K. Dewettinck. 2009. Effect of washing conditions on the recovery of milk fat globule membrane proteins during the isolation of milk fat globule membrane from milk. J. Dairy Sci. 92:3592-3603.

Lee, D. K., J. Ahn, and H. S. Kwak. 1999. Cholesterol removal from homogenized milk with $\beta$-cyclodextrin. J. Dairy Sci. 82:23272330.

Lopez, C., V. Briard-Bion, O. Ménard, E. Beaucher, F. Rousseau, J. Fauquant, N. Leconte, and B. Robert. 2011. Fat globules selected from whole milk according to their size: Different compositions and structure of the biomembrane, revealing sphingomyelin-rich domains. Food Chem. 125:355-368.

Lopez, C., V. Briard-Bion, O. Menard, F. Rousseau, P. Pradel, and J.-M. Besle. 2008. Phospholipid, sphingolipid, and fatty acid compositions of the milk fat globule membrane are modified by diet. J. Agric. Food Chem. 56:5226-5236.

Lopez, C., M.-N. Madec, and R. Jimenez-Flores. 2010. Lipid rafts in the bovine milk fat globule membrane revealed by the lateral segregation of phospholipids and heterogeneous distribution of glycoproteins. Food Chem. 120:22-33.

MacGibbon, A. K. H., and M. W. Taylor. 2006. Composition and structure of bovine milk lipids. Pages 1-42 in Advanced Dairy Chemistry. Vol. 2: Lipids. P. F. Fox and P. L. H. McSweeney, ed. Springer, New York, NY. 
Mather, I. H. 2000. A review and proposed nomenclature for major proteins of the milk-fat globule membrane. J. Dairy Sci. 83:203247.

Mather, I. H. 2011. Milk lipids: Milk fat globule membrane. Pages 680-690 in Encyclopedia of Dairy Sciences. 2nd ed. J. W. Fuquay, ed. Academic Press, San Diego, CA.

McClements, D., E. Decker, Y. Park, and J. Weiss. 2008. Designing food structure to control stability, digestion, release and absorption of lipophilic food components. Food Biophys. 3:219-228.

McMullen, T. P. W., R. N. A. H. Lewis, and R. N. McElhaney. 2004. Cholesterol-phospholipid interactions, the liquid-ordered phase and lipid rafts in model and biological membranes. Curr. Opin. Colloid Interface Sci. 8:459-468.

Michalski, M. C., V. Briard, and F. Michel. 2001. Optical parameters of milk fat globules for laser light scattering measurements. Lait 81:787-796.

Michalski, M.-C., and C. Januel. 2006. Does homogenization affect the human health properties of cow's milk? Trends Food Sci. Technol. $17: 423-437$.

Michalski, M.-C., F. Michel, D. Sainmont, and V. Briard. 2002. Apparent $\zeta$-potential as a tool to assess mechanical damages to the milk fat globule membrane. Colloids Surf. B Biointerfaces 23:23-30.

Op den Kamp, J. A. F. 1979. Lipid asymmetry in membranes. Annu. Rev. Biochem. 48:47-71.

Patton, S., and G. Huston. 1986. A method for isolation of milk fat globules. Lipids 21:170-174.

Ramalho, H. M. M., S. Casal, and M. Oliveira. 2011. Total cholesterol and desmosterol contents in raw, UHT, infant formula powder and human milks determined by a new fast micro-HPLC method. Food Anal. Meth. 4:424-430.
Rombaut, R., J. V. Camp, and K. Dewettinck. 2005. Analysis of phospho- and sphingolipids in dairy products by a new HPLC method. J. Dairy Sci. 88:482-488.

Rombaut, R., J. V. Camp, and K. Dewettinck. 2006. Phospho- and sphingolipid distribution during processing of milk, butter and whey. Int. J. Food Sci. Technol. 41:435-443.

Simons, K., and W. L. C. Vaz. 2004. Model systems, lipid rafts, and cell membranes. Annu. Rev. Biophys. Biomol. Struct. 33:269-295.

Vist, M. R., and J. H. Davis. 1990. Phase-equilibria of cholesterol dipalmitoylphosphatidylcholine mixtures: ${ }^{2} \mathrm{H}$ Nuclear magneticresonance and differential scanning calorimetry. Biochemistry 29:451-464.

Viturro, E., H. H. Meyer, C. Gissel, and M. Kaske. 2010. Rapid method for cholesterol analysis in bovine milk and options for applications. J. Dairy Res. 77:85-89.

Walstra, P. 1999. Dairy Technology: Principles of Milk Properties and Processes. Taylor \& Francis, Boca Raton, FL.

Walstra, P., J. T. M. Wouters, and T. J. Geurts. 2006. Colloidal particles of milk. Pages 127-130 in Food Science and Technology. Vol. 146. CRC/Taylor \& Francis, Boca Raton, FL.

Ye, A., H. Singh, M. W. Taylor, and S. Anema. 2002. Characterization of protein components of natural and heat-treated milk fat globule membranes. Int. Dairy J. 12:393-402.

Zheng, H., R. Jiménez-Flores, and D. W. Everett. 2013. Bovine milk fat globule membrane proteins are affected by centrifugal washing processes. J. Agric. Food Chem. 61:8403-8411.

Zheng, H., R. Jiménez-Flores, D. Gragson, and D. W. Everett. 2014 Phospholipid architecture of the bovine milk fat globule membrane using giant unilamellar vesicles as a model. J. Agric. Food Chem. 62:3236-3243. 